\title{
PLANIFICACIÓN ESTRATÉGICA CORPORATIVA EN PYMES. CASO DE ESTUDIO DE UNA PYME EN MANTA, ECUADOR
}

\section{CORPORATE STRATEGIC PLANNING IN SMEs. CASE STUDY OF A SME IN MANTA, ECUADOR}

\author{
Maldonado-Escandón Andrea ${ }^{1 *}$; Patiño-Patiño Silvia ${ }^{2}$; Chiriboga-Mendoza Fidel ${ }^{3}$ \\ ${ }^{1}$ Universidad Laica Eloy Alfaro de Manabí, ULEAM. Manta, Ecuador. \\ 2Universidad Laica Eloy Alfaro de Manabí, ULEAM. Manta, Ecuador. \\ ${ }^{3}$ Docente de la Universidad Laica Eloy Alfaro de Manabí, ULEAM. Manta, Ecuador.
}

*Correo: ancemes-25@gmail.com

\begin{abstract}
Resumen
La planificación es una herramienta administrativa que permite proyectarse al futuro a través de objetivos y estrategias en una organización. Por los diversos cambios que surgen ya sean estos por efecto político, tecnológico, social o económico es fundamental que la administración se encuentre preparada para responder a estos factores. En el presente trabajo de investigación se analiza el caso de una empresa dedicada a la compra y venta de materiales, suministros eléctricos al por menor, por lo cual este proyecto de investigación mantiene como objetivo conocer la gestión financiera con respecto a la planificación estratégica corporativa, evaluando el entorno, riesgos y oportunidades a través de la aplicación de una herramienta estratégica que permita optimizar los recursos que posee la entidad. Entre los métodos de investigación aplicados son deductivo, cualitativo y cuantitativo, mientras que las técnicas utilizadas se basaron en observación, encuesta y entrevista. Este documento se ha realizado a partir de un enfoque integrador que considere la percepción de los trabajadores, así como del máximo representante de la empresa, para de esta forma analizar el contexto situacional de la pyme y determinar la importancia de la planificación estratégica.
\end{abstract}

Palabras clave: gestión financiera, planificación estratégica, corporación, estrategia.

\begin{abstract}
Planning is an administrative tool that allows projecting into the future through objectives and strategies in an organization. Due to the various changes that arise, whether due to political, technological, social or economic effects, it is essential that the administration is prepared to respond to these factors. In the present research work the case of a company dedicated to the purchase and sale of materials, retail electrical supplies is analyzed, for which reason this research project maintains the objective of knowing financial management with respect to corporate strategic planning, evaluating the environment, risks and opportunities through the application of a strategic tool that enables the entity's resources to be optimized. Among the applied research methods are deductive, qualitative and quantitative, while the techniques used were based on observation, survey and interview. This document has been produced from an integrative approach that considers the perception of the workers, as well as the highest representative of the company, in order to analyze the situational context of the SME and determine the importance of strategic planning.
\end{abstract}

Keywords: financial management, strategic planning, corporation, strategy.

Información del manuscrito:

Fecha de recepción: 18 de septiembre de 2018

Fecha de aceptación: 10 de diciembre de 2018

Fecha de publicación: 10 de enero de 2019 


\section{Introducción}

En los avances financieros de los últimos años se ha presentado un impulso considerable en lo que respecta a la gestión financiera, constituyéndose en una herramienta que permite optimizar recursos, en el trabajo propuesto por CansingMuñoz \& De La Ese (2016) se propuso un plan estratégico para optimizar la gestión financiera de la empresa fabricadora de Conos "Campeón S.A." En donde la problemática quedo definida en determinar las estrategias de aplicación en la gestión financiera que genere la creación de valor y la maximización de la utilidad en pequeñas y medianas empresas.

Además, la influencia de la contaduría pública en la gestión financiera de las organizaciones se evidencia en el ejercicio de auditorías que aportan a través del análisis y evaluación de procedimientos, un análisis de la situación financiera de la organización, determinando de esta forma los factores que potencian el crecimiento del valor (GutiérrezZarabanda, 2013).

El sector económico de las Pymes se encuentra expuesto a limitaciones en su crecimiento, en muchos casos por no contar con las herramientas y estrategias adecuadas para resolver inconvenientes, aplicando técnicas como encuestas, entrevistas y fichas de observación mediante métodos inductivos para investigar los hechos y proponiendo un diseño de plan estratégico para mejorar el funcionamiento de Pymes. Se observa que la aplicación de herramientas financieras es fundamental en una entidad, tal como lo demuestra Carló Del Vecchio (2018).

Luna-Carreño (2016) realizó un diseño de plan estratégico para mejorar la gestión administrativa, financiera y operativa de una empresa de muebles, ubicada en Cuenca, Ecuador, manifestando en la problemática que la entidad requería de un análisis minucioso en todas las áreas para poder ofrecer un diagnóstico y adoptar estrategias que permitieran mejorar su gestión administrativa, financiera $y$ operativa.

Es necesario que las entidades mantengan un aprendizaje respecto a su organización, debido a que según Castaño-Ramírez (2011), la práctica de una planificación 
estratégica adquiere vital importancia para la supervivencia de las organizaciones, haciendo énfasis en que ciertos conceptos clave como organización, entorno y estrategia, no han sido considerados en el manejo de las instituciones. Es por esta razón que el objetivo del presente trabajo es evaluar el contexto y estado actual de la planificación estratégica de una pyme de la ciudad de Manta, Manabí, Ecuador relacionada con el sector de la comercialización de insumos y materiales eléctricos.

\section{Metodología}

La modalidad de investigación aplicada en este proyecto se fundamenta en un trabajo de campo, puesto que para la realización del mencionado proyecto se realizaron visitas en las instalaciones de una pyme que tiene como razón social la comercialización (compra y venta) de materiales de electricidad, ubicada en la ciudad de Manta, durante el período 2016-2017, en el cual se obtuvo la información necesaria para determinar las causas y el respectivo análisis de la problemática.

La investigación realizada es de tipo descriptivo-deductivo, utilizadas con el fin de abordar la problemática de la entidad en función del plan estratégico corporativo.

\subsection{Técnicas e instrumentos}

Las técnicas aplicadas en este trabajo se fundamentan en entrevistas y encuestas. Se realizó una entrevista al representante legal de la pyme en la ciudad de Manta, quien se encuentra vinculado a la empresa, realizando una pregunta abierta para conocer la influencia que tiene la planificación estratégica corporativa. Además, se realizaron encuestas a partir de un banco de preguntas cerradas organizadas, dirigidas al personal administrativo y operativo de una pyme que tiene como razón social la comercialización (compra y venta) de materiales de electricidad, con el fin de presentar las respuestas $u$ opiniones de los mismos.

\subsection{Población y muestra}

En la presente investigación se consideró una población de 100 personas, tomando en cuenta las áreas administrativas y operativas de la empresa pyme mantense.

El estudio se realizó considerando el $100 \%$ de la población. 


\section{Resultados y discusión}

La entrevista realizada al gerente general de la pyme determinó que la empresa no planifica estrategias, puesto que responde a cambios de precios de

acuerdo a la competencia. El informante manifiesta que "tengo colegas que son proveedores de materiales y siempre converso con ellos para saber qué precios han subido y cuales han bajado o se encuentran en ofertas". Con respecto a la gestión financiera se pretende llevar el mejor orden en el efectivo, cada uno de los socios mantiene su sueldo y se evita desviar recursos, que en determinados momentos no serían una inversión para la empresa. "Considero que el tema económico ha generado no solo a mí, sino algunos empresarios 0 emprendedores que disminuyan las ventas, en nuestro caso comparando este año con el año pasado (2017) si existen cambios, los ingresos no son los mismos, al inicio del año se redujo personal y hoy día por el contacto que tengo con ellos, se los contrata eventualmente".

Con respecto al personal administrativo y operativo, el $55 \%$ de los 100 participantes, afirma que una vez al año reciben socialización de los procedimientos de gestión financiera, el $40 \%$ que nunca lo han realizado y $5 \%$ indica que una vez al mes. Con respecto al personal administrativo y operativo el $61 \%$ no conoce nada sobre la misión, visión, valores corporativos y objetivos de la empresa, un $37 \%$ que muy poco y un $2 \%$ afirma que sí conocen. Se evidencia que existe un desconocimiento masivo de la misión, visión y los valores comparativos de la organización.

Cabe indicar que, respecto a las planificaciones estratégicas, el $62 \%$ del personal administrativo y operativo afirma que nunca lo hace, un $35 \%$ a veces y el $3 \%$ que siempre lo realiza.

Referente a la encuesta aplicada, el mayor porcentaje $(79 \%)$ afirma que la empresa no realiza un FODA estratégico. Por su parte, el $80 \%$ indica que desde la alta dirección de la organización no se realiza la evaluación mediante las fuerzas de PORTER. Un comportamiento similar se observa en la estrategia PEST, debido a que el $82 \%$ de los participantes manifiesta que nunca se ha aplicado esta estrategia. 
Del análisis de la investigación realizada se deducen las situaciones positivas, obteniendo como resultado que la empresa socializa sus procedimientos de gestión financiera por lo menos una vez en el año, adicional a ello el personal manifestó que dichos procedimientos podrían calificarse como buenos, sin embargo, se pueden mencionar aspectos negativos referentes a que el personal no conoce acerca de la misión, visión, objetivos y valores corporativos, además, no ha aplicado herramientas de gestión para evaluar su entorno. Maldonado \& Erazo (2016) señalan que los factores fundamentales que inciden en la planificación estratégica de las pymes son la falta de procedimientos formales de planificación estratégica, la forma específica en la que operan las pymes en base a decisiones y proyectos específicos sobre los cuales definen estrategias a largo plazo en función de parámetros como calidad, estabilidad y mejora continua en las actividades del talento humano, puesto que puede generarse un distanciamiento entre los requerimientos del mercado laboral y la capacitación y formación en las pymes.

Adicionalmente, se resalta la importancia de la planificación estratégica; según Ogliastri (1999) el fin de la planificación estratégica es propiciar un desarrollo sostenible en las organizaciones que permita coordinar las actividades internas de la organización a partir de criterios y procedimientos estandarizados que solucionen los conflictos de interés en función de los objetivos y metodologías diseñadas para el cumplimiento de estos. Cabe señalar que el caso de estudio evaluado determina una realidad que es común en gran parte de las pymes de Latinoamérica, debido a que se subestima la importancia de la gestión organizada y la planificación estratégica. De conformidad con lo antes mencionado, el proceso de planificación genera un direccionamiento correcto $y$ un ambiente óptimo para la gestión empresarial basada en principios de innovación y mejora continua.

A continuación, se realiza un análisis FODA de la pyme evaluada (fortalezas, oportunidades, debilidades y amenazas). 


\subsection{Análisis FODA}

La figura 1 muestra el análisis FODA de la empresa investigada a partir de la aplicación de los instrumentos de investigación.

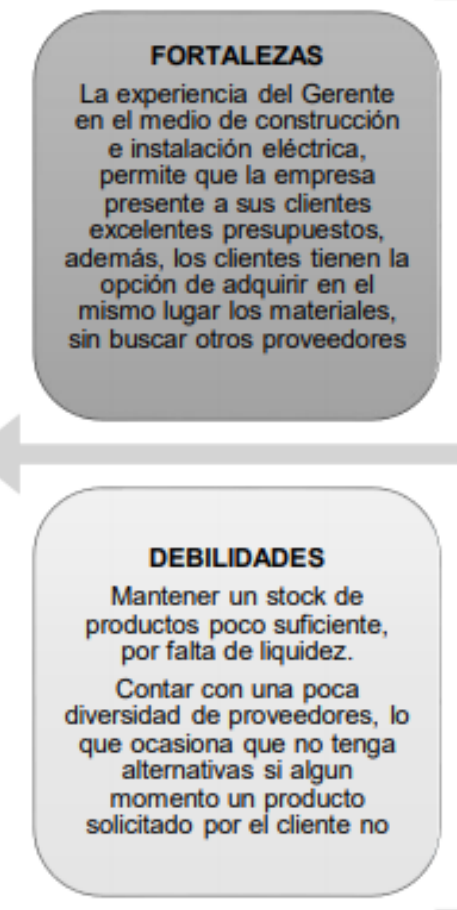

\section{OPORTUNIDADES}

Los trabajadores son de confianza y mantiene la experiencia suficiente

respondiendo con excelencia con o sin supervisión.

Esto permite que la administración contribuya a mejorar los beneficios de estos.

Figura 1. Análisis FODA de la pyme

Sánchez-Quintero (2003) menciona que una adecuada práctica de planificación estratégica considera aspectos como la información sobre la evaluación interna, así como lo que ocurre externamente y puede incidir en el desempeño corporativo o empresarial; por otra parte, la formulación de una adecuada misión, visión y objetivos acompañados de análisis situacionales o auditorías para aplicar los principios de mejora continua.

\section{Conclusiones}

La gestión financiera cumple un papel muy importante en las organizaciones; a través de la aplicabilidad de la planificación estratégica corporativa la entidad además de planificar, puede reducir recursos, debido a que las herramientas estratégicas permiten evaluar el evaluar el entorno. Por lo 
tanto, se aplicó un FODA, como herramienta para evaluar los factores internos y externos de la pyme analizada en el presente caso de estudio.

La omisión de la planificación estratégica corporativa en una empresa conlleva a que se ignoren los cambios en su entorno, y no se actualicen las técnicas y estrategias para sobrellevar los cambios relacionados con macro y microeconomía.

Se resalta la importancia de la brecha que existe entre las pymes y las grandes empresas en cuanto a la racionalidad. Este parámetro es entendido como la coherencia en las acciones y estrategias aplicadas para alcanzar unos objetivos determinados. Por lo tanto, uno de los factores que influye en el posicionamiento de una pyme, como la que se analiza en este caso de estudio, es la adecuada relación entre los fines y medios empleados.

\section{Bibliografía}

Cansing-Muñoz, A., \& De La Ese Canales, M. E. (2016). Plan Estratégico para optimizar la gestión financiera de la Empresa Fábrica de Conos Campeón S.A. Guayaquil, Ecuador.
Carló Del Vecchio, T. (2018). Análisis de la planificación estratégica de las mipymes en la ciudad de Esmeraldas. Tesis de pregrado PUCESE, Esmeraldas. Obtenido de https://repositorio.pucese.edu .ec/handle/123456789/1486

Castaño-Ramírez, A. (2011). Planeación estratégica en la Pyme de Bogotá. Orientación al futuro y evasión de la incertidumbre. Contaduría y administración, (235), 147173.

Gutiérrez-Zarabanda, A. Y. (2013). El contador público dentro de la Gestión Financiera. Bogotá, Colombia. Obtenido de https://repository.unimilitar.ed u.co/.../1/GutiérrezZarabanda AlecyYovana2013.pd

Luna-Carreño, H. (2016). Diseño de un plan estratégico para mejorar la gestión administrativa, financiera, y operativa de la empresa de muebles Cllassic, ubicada en la ciudad Cuenca provincia del Azuay. Tesis de pregrado. Universidad Laica Vicente Rocafuerte de Guayaquil, Ecuador. Obtenido de http://repositorio.ulvr.edu.ec/h andle/44000/722

Maldonado, G. V., \& Erazo, M. A. (2016). El reto de la planificación estratégica en las Pymes. Revista publicando, 3(8), 335-344. 
Ogliastri, E. (1990). Manual de planeación estratégica. Teoría, aplicaciones y casos. Bogotá: Tercer Mundo.
Sánchez-Quintero, J. (2003). Estrategia integral para pymes innovadoras. Revista Escuela de Administración de Negocios, (47), 35-45 